\title{
Risk Aggregation by Using Copulas in Internal Models
}

\author{
Tristan Nguyen, Robert Danilo Molinari \\ Department of Economics, WHL Graduate School of Business and Economics, Lahr, Germany \\ E-mail:tristan.nguyen@whl-lahr.de \\ Received August 17, 2011; revised October 9, 2011; accepted October 20, 2011
}

\begin{abstract}
According to the Solvency II directive the Solvency Capital Requirement (SCR) corresponds to the economic capital needed to limit the probability of ruin to $0.5 \%$. This implies that (re-)insurance undertakings will have to identify their overall loss distributions. The standard approach of the mentioned Solvency II directive proposes the use of a correlation matrix for the aggregation of the single so-called risk modules respectively sub-modules. In our paper we will analyze the method of risk aggregation via the proposed application of correlations. We will find serious weaknesses, particularly concerning the recognition of extreme events, e.g. natural disasters, terrorist attacks etc. Even though the concept of copulas is not explicitly mentioned in the directive, there is still a possibility of applying it. It is clear that modeling dependencies with copulas would incur significant costs for smaller companies that might outbalance the resulting more precise picture of the risk situation of the insurer. However, incentives for those companies who use copulas, e.g. reduced solvency capital requirements compared to those who do not use it, could push the deployment of copulas in risk modeling in general.
\end{abstract}

Keywords: Solvency II, Risk Capital, Risk Measures, Risk Dependencies, Aggregation of Risks, Copulas

\section{Introduction}

The Solvency II directive of the European Commission [1] focuses on an economic risk-based approach and therefore obliges insurance undertakings to determine their overall loss distribution function. The increasing complexity of insurance products makes it necessary to consider dependencies between the single types of risk to determine this function properly. Neglecting those dependencies may have serious consequences underestimating the overall risk an insurer is facing. On the other hand, assuming complete dependency between risks may result in an overes timate of capital requirements and therefore incur too high capital costs for an insurance company. The Solvency II draft directive acknowledges this fact and proposes recognition of dependencies by the use of linear correlations. Reason is that correlations are relatively easy to understand and to apply. However, the use of correlation requires certain distributional assumptions which are invalidated e.g. by non-linear derivative products and the typical skew and heavy tailed insurance claims data. Therefore aggregation of insurance risks via correlations may neglect important information concerning the tail of a distribution.

In contrast, copulas provide full information of de- pendencies between single risks. Therefore they have become popular in recent years. The copulas concept in an insurance context was first introduced by Wang [2], who discusses models and algorithms for the aggregation of correlated risk portfolios. Frees and Valdez [3] provided an introduction to the use of copulas in risk measurement by describing the basic properties of copulas, their relationships to measures of dependence and several families of copulas. Blum, Dias and Embrechts [4] discuss the use of copulas to handle the measurement of dependence in alternative risk transfer products. McNeil [5] presents algorithms for sampling from a specific copula class which can be used for higher-dimensional problems. Eling and Toplek [6] analyze the influence of non-linear dependencies on a non-life insurer's risk and return profile.

As copulas allow the separate modeling of risks and the dependencies between them, it will also be possible to explore the impact of (different) dependency structures on the required solvency capital if they are used [7]. Different dependency structures can be modeled on the one hand through modified parameters of the copula function and on the other hand through the choice of a completely different copula family. Following some recent contributions $[8,9]$, our aim is to give an overview over the concept of copulas, to analyze and discuss their possible application 
in the context of Solvency II and finally to make them accessible to a wider circle of users. In this context we would also like to discuss, if the new Solvency II directive forms an accurate concept for considering risk dependencies or if further adjustments should be made. Relating to that it will also be necessary to discuss dependency ratios like the correlation coefficient but also others (e.g. Spearman's rank correlation).

We will therefore start with an overview over dependency ratios. In Section 3 we will continue with the introduction of copulas and illustrate different families and types of copulas. After that we will briefly describe how copulas and multivariate distributions can be determined out of empirical data in Section 4. The paper will continue with an assessment of the presented dependence concepts in Section 5 and end with a description and an assessment of the consideration of risk dependencies in the Solvency II framework in Section 6.

\section{Dependency Ratios}

Using the linear correlation coefficient is a very rudimentary, but also simple way of describing risk dependencies in a single number. The linear correlation coefficient of real valued non-degenerate random variables $X$, $Y$ is defined by the following Equation (1):

$$
\rho(X, Y)=\frac{\operatorname{Cov}(X, Y)}{\sqrt{\operatorname{Var}(X) \times \operatorname{Var}(Y)}},
$$

where $\rho(X, Y)$ is the linear correlation coefficient of $X$ and $Y, \operatorname{Cov}(X, Y)=E[X Y]-E[X] E[Y]$ is the covariance of $X$ and $Y$ and $\operatorname{Var}(X)$ and $\operatorname{Var}(Y)$ are the finite variances of $X$ and $Y$.

In case of multiple dimensions the so called correlation matrix needs to be applied. Equation (2) shows this symmetric and positive semi-definite correlation matrix:

$$
\begin{array}{rlcc}
\rho\left(X_{1}, Y_{1}\right) & \cdots & \rho\left(X_{1}, Y_{n}\right) \\
\vdots & \ddots & \vdots \\
\rho\left(X_{n}, Y_{1}\right) & \cdots & \rho\left(X_{n}, Y_{n}\right) \\
\text { i.e. } \rho(X, Y)_{a, b}= & \rho\left(X_{a}, Y_{b}\right), 1 \leq \mathrm{a}, \mathrm{b} \leq \mathrm{n} .
\end{array}
$$

The linear correlation coefficient (also called Pearson's linear correlation) measures only linear stochastic dependency of two random variables. It takes values between -1 and 1 , i.e. $-1 \leq \rho(X, Y) \leq+1$. However, perfectly positive correlated random variables do not necessarily feature a linear correlation coefficient of 1 and perfectly negative correlated random variables do not necessarily feature a linear correlation coefficient of -1 . Random variables that are strongly dependent may also feature a linear correlation coefficient which is according to amount close to 0 .

Two pairs of random variables with a certain linear correlation coefficient may actually have a completely different dependence structure. Figure 1 which shows realizations of two pairs of random variables $\left(X_{1}\right.$ and $X_{2}$ respectively $X_{1}$ and $\left.X_{3}\right)$ that both have the same linear correlation, clearly illustrates this.

The covariance and thus also the linear correlation between independent random variables is zero. However, if the linear correlation coefficient between two random variables is near zero, it can actually exist a high correlation between them. Linear correlation is a natural dependency ratio for elliptically distributed risks. If used for random variables that are not distributed elliptically, linear correlation can lead to wrong results. Extreme events with high losses can be severely underestimated by using the linear correlation as a measure for dependencies between risks [10]. Modeling major claims often requires the use of distributions with infinite variances for which the linear correlation coefficient is not defined. In addition linear correlation is not invariant concerning non-linear monotone transformations which may cause problems when an amount of loss is converted into a loss payment.

Two other ratios for measuring risk dependencies are e.g. Spearman's rank correlation and Kendall's $\tau$. However, they do also not fully inform on dependencies between risks, but rather compress all information into a single number. The coefficient of tail dependence (important for non-life insurers modeling extreme events) for two random variables $X$ and $Y$ describes the likelihood of $Y$ taking an extreme value on condition that $X$ also takes an extreme value. This also means that the coefficient of tail dependence does not provide full information on the dependence structure between random variables. The following Equations (3)-(5) show the three dependency ratios:

$$
\rho_{S}(X, Y)=\rho\left(F_{X}(X), F_{Y}(Y)\right)
$$

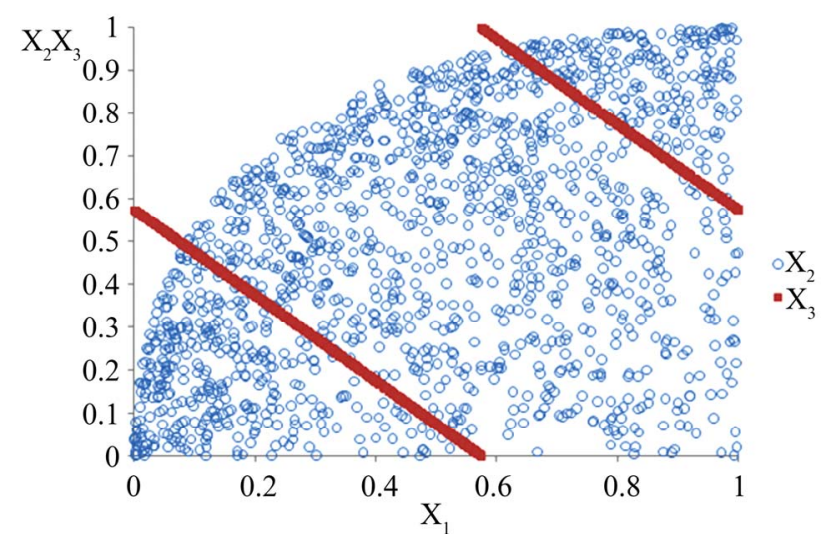

Figure 1. Dependence structure between random variables $X_{1}$ and $X_{2}$ respectively $X_{1}$ and $X_{3}[11]$. 
where $F_{x}$ is the distribution function of $X, F_{y}$ is the distribution function of $Y$ and $F$ would be the joint distribution function.

$$
\begin{aligned}
\tau(X, Y)= & P\left(\left(X_{1}-X_{2}\right)\left(Y_{1}-Y_{2}\right)>0\right) \\
& -P\left(\left(X_{1}-X_{2}\right)\left(Y_{1}-Y_{2}\right)<0\right)
\end{aligned}
$$

where $\left(X_{1}, Y_{1}\right)$ and $\left(X_{2}, Y_{2}\right)$ are two independent and identically distributed pairs of random variables from $F$.

$$
\lambda(X, Y)=\lim _{\alpha \rightarrow 1-} P\left(Y>F_{Y}^{-1}(\alpha) \mid X>F_{X}^{-1}(\alpha)\right),
$$

on the condition that this limit $\lambda \in[0,1]$ exists.

\section{Copulas}

In contrast, copulas provide full information on the dependency structure between risks. Copulas allow the separation of the joint marginal distribution function into a part that describes the dependence structure and parts that describe the marginal distribution functions.

The Copula is a multivariate distribution function with margins that are uniformly distributed on $[0,1]$ and was defined by Sklar [12]:

$$
C\left(u_{1}, \cdots, u_{n}\right)=P\left(U_{1} \leq u_{1}, \cdots, U_{n} \leq u_{n}\right),
$$

where $C()$ is the copula, $\left(U_{1}, \cdots, U_{n}\right)^{T}$ with $U_{i} \sim U(0,1)$ for all $i=1, \cdots, n$ a vector of random variables and $\left(u_{1}, \cdots, u_{n}\right)^{T} \in[0,1]^{n}$ realizations of $\left(U_{l}, \cdots, U_{n}\right)^{T}$.

The risk modeling process with copulas consists of two steps. First one has to determine the marginal distribution of every single risk component. Secondly the dependence structure between these risk components has to be determined via the copula function. In order to obtain the joint distribution function the $n$ single risks $X_{i}$ have then to be transformed each into a random variable $U_{i}$ that is uniformly distributed on $[0,1]$ by using using the corresponding marginal distribution $F_{i}$

$$
U_{i}=F_{i}\left(X_{i}\right) \text {. }
$$

We obtain the multivariate distribution function by inserting these transformed random variables into the copula function:

$$
F\left(x_{1}, \cdots, x_{n}\right)=C\left(u_{1}, \cdots, u_{n}\right)=C\left(F_{1}\left(x_{1}\right), \cdots, F_{n}\left(x_{n}\right)\right)(8)
$$

In case of continuous and differentiable marginal distributions and a differentiable copula the joint density is:

$$
f\left(x_{1}, \cdots, x_{n}\right)=f_{1}\left(x_{1}\right) * \ldots * f_{n}\left(x_{n}\right) * c\left(F_{1}\left(x_{1}\right), \cdots, F_{n}\left(x_{n}\right)\right) \text {, }
$$

where $f_{i}\left(x_{i}\right)$ is the respective density for distribution function $F_{i}$ and

$$
c\left(u_{1}, \cdots, u_{n}\right)=\frac{\partial^{n} C\left(u_{1}, \cdots, u_{n}\right)}{\partial u_{1} \cdots \partial u_{n}}
$$

the density of the copula.

In this way we can derive a multivariate distribution function out of specified marginal distributions and a copula that contains information about the dependence structure between the single variables. But also the opposite holds: A copula can be determined out of the inverse of the marginal distributions and the multivariate distribution function.

The most important copula families are (the bands in which the dependencies are stronger or weaker differ):

- $\quad$ Elliptical copulas

o Gaussian copulas

o Student copulas

- Archimedean copulas

o Gumbel copulas

o Cook-Johnson copulas

o Frank copulas

Equation (10) shows the definition of the Gaussian copula:

$$
C_{\rho}^{\text {Gau }}\left(u_{1}, \cdots, u_{n}\right)=\Phi_{\rho}^{n}\left(\Phi^{-1}\left(u_{1}\right), \cdots, \Phi^{-1}\left(u_{n}\right)\right),
$$

$\Phi_{\rho}^{n}$ is the distribution function of the n-variate standard normal distribution with correlation matrix $\rho$ and $\Phi^{-1}$ is the inverse of the distribution function of the univariate standard normal distribution. The dependency in the tails of multivariate distributions with a Gaussian copula goes to zero [13], which means that the single random variables of the joint distribution function are almost independent in case of high realizations. Insurance risks feature in most cases weak dependency for lower values and strong dependency for higher realizetions. From this perspective the Gaussian copula does not provide a proper basis for modeling insurance risks.

In contrast, Student copulas do not feature independency in the tails of a distribution [7]. Equation (11) shows how they can be defined:

$$
C_{v, \rho}^{S t u}\left(u_{1}, \cdots, u_{n}\right)=t_{v}^{n}\left(t_{v, \rho}^{-1}\left(u_{1}\right), \cdots, t_{v}^{-1}\left(u_{n}\right)\right),
$$

where $v$ is the number of degrees of freedom, $t_{v, \rho}^{n}$ the distribution function of the $n$-variate Student distribution with $v$ degrees of freedom and a correlation matrix of $\rho$ and $t_{v}^{-1}$ the inverse of the distribution function of the univariate Student distribution with $v$ degrees of freedom.

The Figures 2 and $\mathbf{3}$ show the densities for both the bivariate Gaussian and the bivariate Student copula. Another class of copulas is given by the Archimedean copulas which can be written in the form:

$$
C\left(u_{1}, \cdots, u_{n}\right)=\phi^{-1}\left(\phi\left(u_{1}\right)+\cdots+\phi\left(u_{n}\right)\right)
$$

for all $0 \leq u_{1}, \cdots, u_{n} \leq 1$ and $\phi$ is some continuous function (called the generator) satisfying: 


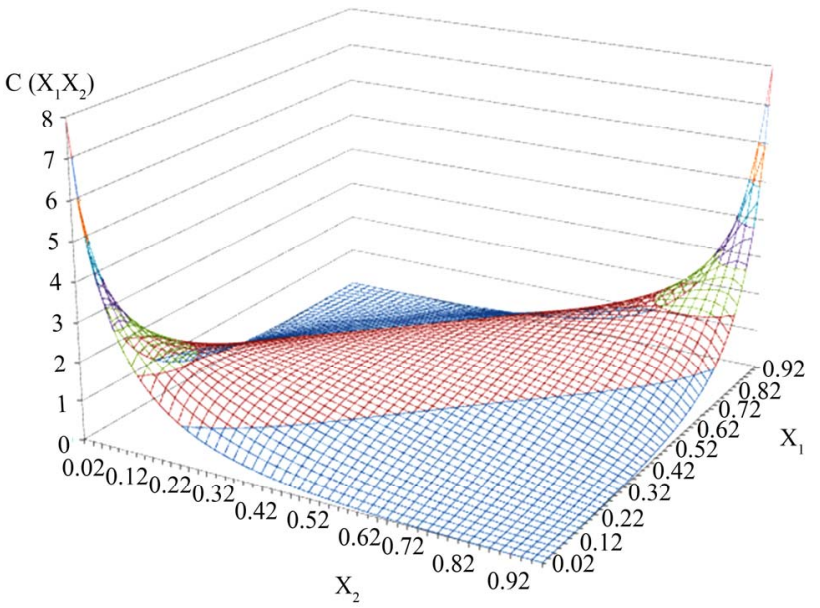

Figure 2. Density of a Gaussian copula (correlation $\rho$ of 0.7 ).

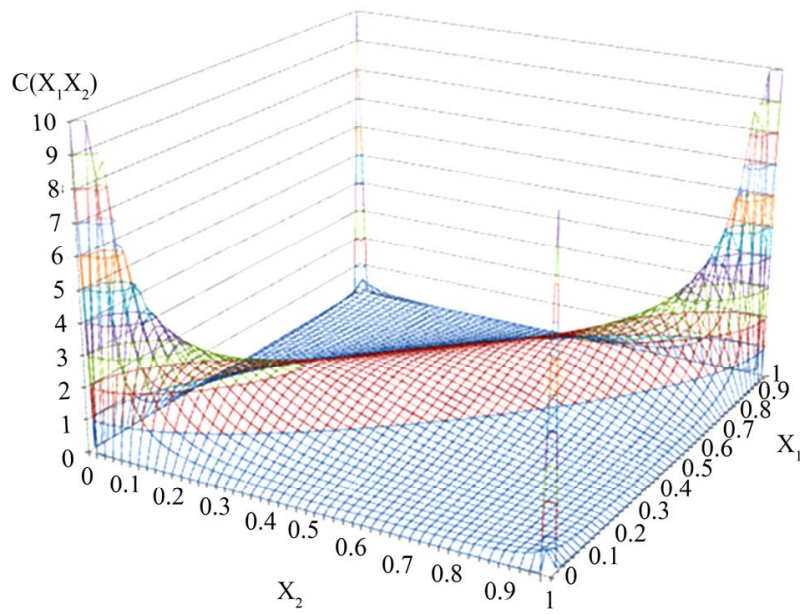

Figure 3. Density of a student copula (correlation $\rho$ of 0.7 ).

1) $\phi(1)=0$;

2) $\phi$ is strictly decreasing and convex and;

3) $\phi^{-1}$ is completely monotonic on $[0, \infty)$.

Among others the Gumbel copulas belong to the Archimedean copulas. Similarly to the Student copulas they are tail dependent, however, not in both the upper and the lower tail, but only in the upper one (see Figure 4).

Therefore they are adequate for modeling extreme events: On the one hand stress scenarios [14] (with high losses and high dependence) can be captured and on the other hand common (lower) losses which in general appear independent can be modeled. Equation (13) shows the formula for Gumbel copulas:

$$
C_{\beta}^{\text {Gum }}\left(u_{1}, \ldots, u_{n}\right)=\mathrm{e}^{\left(-\left(\sum_{i=1}^{n}\left(-\ln \left(u_{i}\right)\right)^{\beta}\right)^{\frac{1}{\beta}}\right)},
$$

$\beta \geq 1$ is a structural parameter. $\beta=1$ leads to a multivariate distribution of independent random variables. Only in this case the Gumbel copula is independent in the upper tail.

Cook-Johnson copulas represent another Archimedean copula. Contrary to the Gumbel copulas they are tail dependent only in the lower tail (see Figure 5). Therefore they perform good results if used for modeling yields on shares [15]. The following equation describes the Cook Johnson copulas:

$$
C_{\beta}^{C-J}\left(u_{1}, \cdots, u_{n}\right)=\left(u_{1}^{-\beta}+\cdots+u_{n}^{-\beta}-n+1\right)^{-\frac{1}{\beta}},
$$

$\beta>0$ is a structural parameter.

The third type of Archimedean copulas presented in this paper is the Frank copula. This type of copulas is completely tail independent [16,17]. The dependence structure given by a copula of this type is similar to one represented by a Normal copula even though the dependence

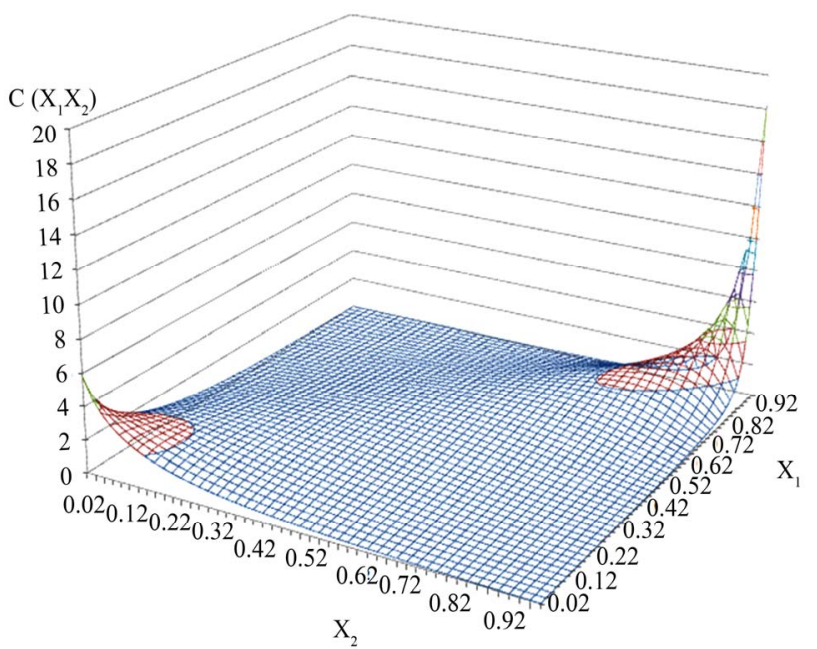

Figure 4. Gumbel copula with structural parameter $\beta=2$.

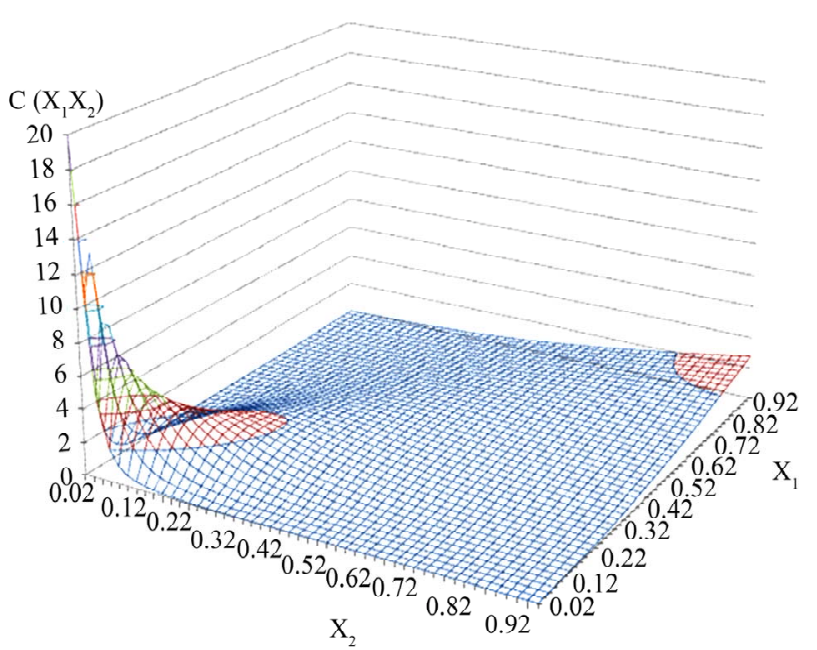

Figure 5. Cook-Johnson copula with structural parameter $\beta=2$. 
in the tail is even lower (see Figure 6). Equation (15) shows the definition of Frank copulas:

$$
C_{\beta}^{F r a}\left(u_{1}, \cdots, u_{n}\right)=-\frac{1}{\beta} \ln \left(1+\frac{\left(\mathrm{e}^{-\beta u_{1}}-1\right) \cdots\left(\mathrm{e}^{-\beta u_{n}}-1\right)}{\left(\mathrm{e}^{-\beta}-1\right)^{n-1}}\right),
$$

$\beta>0$ is a structural parameter.

\section{Determination of Copulas and Multivariate Distributions}

Using the concept of copulas for capturing dependencies between risks in an insurance company, first of all the corresponding copulas have to be determined. Two alternative approaches for achieving this are parametric and non-parametric approaches. Using a parametric approach means to first determine the respective type of copula [10]. As shown above the various types of copulas describe a different type of dependence structure each. Therefore it is necessary to choose that type of copulas that best fits the actual dependence structure. We can follow a procedure for the bivariate case established by Genest and Rivest [18] which uses the dependency ratios for identifying a type of Archimedean copula that fits the observations. The procedure is carried out in 3 steps [19]:

1) Estimation of Kendall's $\tau$ out of the observations $\left(X_{11}, X_{21}\right), \cdots,\left(X_{1 n}, X_{2 n}\right)$.

2) Define an intermediate random variable $Z_{i}=F\left(X_{1 i}\right.$, $X_{2 i}$ ) with distribution function K. Genest, C. and Rivest, L.-P. (1993) showed that the following statement holds:

$$
K(z)=z-\frac{\phi(z)}{\phi^{\prime}(z)}
$$

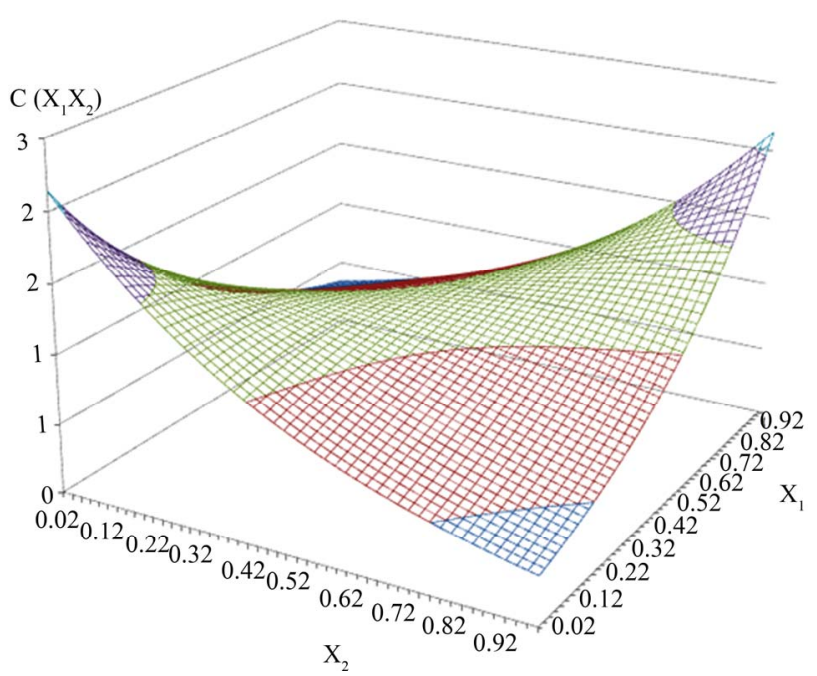

Figure 6. Frank copula with structural parameter $\beta=2$. $\phi(z)$ is the generator of (and therefore determines) an Archimedean copula.

Construct a non-parametric estimate of $K$ :

a) Define pseudo observations $Z_{i}=$ number of $\left(X_{1 j}\right.$, $\left.X_{2 j}\right)$ such that $X_{1 j}<X_{1 i}$ and $\left.X_{2 j}<X_{2 i}\right\} /(\mathrm{n}-1)$ for $i=1, \cdots, \mathrm{n}$.

b) Estimate of $K$ is $K_{n}(z)=$ number of $Z_{i} \leq$ $z\} /\left\{\right.$ number of $\left.Z_{i}\right\}$.

3) Construct parametric estimate of $K$ using the relationship of (16): Use the estimate of Kendall's $\tau$ from Step 0 and the given relation between Kendall's $\tau$ and the generator of a specific type of a copula $\phi(z)$ to come to a parametric estimate of $K$.

Repeat step three with generators for different types of copulas. At the end choose that type of copula where the parametric estimate of $K$ most closely resembles the non-parametric estimate of $K$ calculated in Step 0.

Once the type of copula is chosen, the parameters of the copula have to be determined as a best-estimate. This can be achieved in course of the estimation of the parameters of the marginal distribution by using the maximum likelihood method [16]. Since one of the advantages of using copulas is the separate estimation of the marginal distributions and the dependence structure also a two-step approach can be applied: in the first step the parameters of the marginal distributions are estimated and in a second step those of the copula.

Using the non-parametric approach means determining an empirical copula out of the empirical data and therefore not determining a specific copula type in advance [10].

\section{Assessment}

In the previous sections we provided an overview over dependency ratios and copulas, two - very different-concepts for describing dependencies between risks. Both of these concepts will be assessed in the following. However, first we will introduce 5 criteria that dependency ratios should meet.

\subsection{Criteria for Dependency Ratios}

The following five criteria are desirable for a dependency ratio. Therefore we will first explain the criteria and afterwards match the introduced dependency ratios with them. If $\delta()$ is a dependency ratio, the criteria can be described as the following:

1) symmetry: $\delta(X, Y)=\delta(Y, X)$;

2) standardization: $-1 \leq \delta(X, Y) \leq 1$;

$3)$ conclusion based on and on co- and countermonotonity;

a) $\delta(X, Y)=1 \Leftrightarrow X, Y$ are comonotone;

b) $\delta(X, Y)=-1 \Leftrightarrow X, Y$ are countermonotone; 
4) Invariance with regard to strictly monotone transformations: For a transformation $T: \mathfrak{R} \rightarrow \mathfrak{R}$ strictly monotone on the codomain of $X$ the following holds:

a) $\delta(T(X), Y)=\delta(X, Y)$, if $T$ is strictly monotonic increasing;

b) $\delta(T(X), Y)=-\delta(X, Y)$, if $T$ is strictly monotonic decreasing;

5) conclusion based on and on independence $\delta(X, Y)=0 \Leftrightarrow X, Y$ are independent.

The first criterion is desirable for dependency ratios because otherwise the resulting dependency ratio would depend on the order of the considered risks. If a dependency ratio fulfills the second criterion, this will lead to an unique measure which makes dependencies between pairs of random variables comparable. Conclusion based on and on co- and countermonotonity helps to immediately detect strongly dependent random variables.

Invariance with regard to strictly monotone transformations is mainly important if the dependency ratio is used for practical applications. If a random variable $X$ is transformed into another variable $T(X)$ using a strictly monotone function $T$, the dependence structure between $X$ and a second random variable $Y$ will be the same as the dependence structure between $T(X)$ and $Y$. Therefore also the dependency ratio should take on the same value for $T(X)$ and $Y$ as for $X$ and $Y$. The last criterion makes sure that also independency between random variables can be detected.

\subsection{Assessment of the Introduced Concepts}

First, we want to assess the dependency ratios. The most popular of those - the Pearson linear correlation coefficient - only fulfills the first two criteria of the above mentioned five. From this point of view it is inferior compared to Spearman's rank correlation and Kendall's $\tau$ which fulfill the first four of the mentioned five criteria. Furthermore, the Pearson linear correlation coefficient is defined only if the variances of the random variables are finite.

Another advantage of both Spearman's rank correlation and Kendall's $\tau$ is that they do not only measure the linear dependency between random variables, but also the monotone dependence in common [13]. Their calculation may be sometimes easier, but sometimes more difficult than the calculation of the Pearson linear correlation coefficient. Using e. g. multivariate normal distributions or multivariate Student distributions the calculation of the momentum based linear correlation coefficient is easier. However, if we consider multivariate distributions that have a dependence structure represented by a Gumbel copula, the calculation of Spearman's rank correlation and Kendall's $\tau$ might be easier.

The coefficient of tail dependence introduced in Section 2 should not be compared to one of the above mentioned three dependency ratios, since it focuses only on the dependency in the tails of a distribution. It should therefore be applied if it is required by the respective problem. This is the case mainly if extreme events are modeled. Therefore we think that matching the five criteria with the coefficient of tail dependence is not reasonable.

Copulas can be used to model multivariate distributions which fully describe the dependence structure. In this way a whole picture of the aggregate risk an insurer is facing. The fact that a given copula implies a certain value for the correlation, but in general not the other way around, makes clear that a copula contains much more information than a dependency ratio. Especially, when dependencies are not linear, but are located in the tails, risk could be significantly underestimated if incomplete information is considered.

From a technical point of view copulas offer the opportunity to first model the marginal distribution functions representing the single risks and in a second step modeling the dependence structure independently from the single risks. Furthermore, similar to Spearman's rank correlation and Kendall's $\tau$ copulas are invariant with regard to strictly monotone transformations [11]. A further technical advantage of using copulas instead of directly modeling multivariate distributions is that the marginal distribution function can then be of any type, whereas if the multivariate distribution function was directly modeled, each of the marginal distribution functions would have to be of the same type. Besides, directly modeling the multivariate distribution function presumes that a dependency ratio is given and therefore once more allows only the use of only a ratio as a dependency measurement.

In summary, we can say that the concept of copulas is clearly superior with regard to the quality of estimating dependencies between risks. Even though ratios currently have advantages in their practical usage - if the usage of copulas becomes more popular in future, these advantages of dependency ratios will be likely to disappear.

All in all we can say that since dependency ratios do not provide a complete picture of the actual situation with regard to risk dependencies, therefore provide significant less information and finally may lead to an underestimation of the actual risk of an insurance company, copulas should be used to describe dependencies between risks in an insurance company if possible. Particularly for the option of introducing an internal risk model the application of copulas seems to be suitable.

\section{Solvency II}

\subsection{Consideration of Risk Dependencies in Solvency II}

According to the new European solvency system (Solvency 
II) insurance undertakings will have to determine the so called Solvency Capital Requirement (SCR) which reflects the amount of capital that is necessary to limit the probability of ruin to $0.5 \%$. That implies that they will also have to determine their overall loss distribution function. Hereby at least the following risks have to be considered [1]:

- $\quad$ non-life underwriting risk;

- life underwriting risk;

- $\quad$ health underwriting risk;

- market risk;

- credit risk;

- operational risk.

Insurers will be able to either use a standard approach or to determine the Solvency Capital Requirement or parts thereof by the use of an internal model. In the latter case the internal model has to be approved by the supervisory authorities. Using the standard approach, the SCR is the sum of the Basic Solvency Capital Requirement, the capital requirement for operational risk and the adjustment for the loss-absorbing capacity of technical provisions and deferred taxes.

The Basic Solvency Capital Requirement consists at least of a risk module for non-life underwriting risk, for life underwriting risk, for health underwriting risk, for market risk and for counterparty default risk each. These risk modules have to be split into sub-modules [1]. The sub-modules shall be aggregated using the same approach as for the aggregation of the risk modules that is described in the following.

After having been determined, the risk modules have to be aggregated. The Solvency II directive clearly states that for the standard approach this has to be done by using correlations and the following Equation (17):

$$
B S C R=\sqrt{\sum_{i, j} \rho_{i, j} * S C R_{i} * S C R_{j}},
$$

$B S C R$ is the Basic Solvency Capital Requirement, $S C R_{i}$ respectively $S C R_{j}$ are risk-modules $i$ respectively $j$ and $\rho_{i, j}$ is the correlation between them.

Table 1 shows the correlation matrix for aggregating risk modules in Solvency II, where $i, j=1$ : risk module for market risk, $i, j=2$ : risk module for counterparty default risk, $i, j=3$ : risk module for life underwriting risk, $i, j=4$ : risk module for health underwriting risk, $i, j=5$ : risk module for non-life underwriting risk.

\subsection{Assessment of the Solvency II Rules with Regard to Risk Dependencies}

On a first level the Basic Solvency Capital Requirement, the capital requirement for operational risk and the adjustment for the loss-absorbing capacity of technical provisions and deferred taxes have to be aggregated. This is done simply by adding the capital requirements which assumes that those risks are fully dependent. However, the assumption that full dependence between e.g. the operational risk and the risks covered by the BSCR is not realistic and therefore the result for the SCR will be too high. However, the amount of solvency capital for the operational risk is limited [1]. Against this background we think that it would be sensible to only consider the operational risk qualitatively like Switzerland has decided in the Swiss Solvency Test instead of simply adding an amount of capital to the Basic Solvency Capital Requirement.

The Solvency II framework closely recognizes dependencies at least in the calculation of the BSCR. However, the standard approach uses the concept of linear correlations to consider dependencies between the risk modules and not copulas which is certainly due to the application of the proportionality principle in the Solvency II directive which has to assure that the regulation is not too burdensome for small and medium-sized insurance undertakings.

Another shortcoming of the regulation set is that the given values for the correlations which are shown in Table 1 seem to be highhanded and do not reflect the specific situation of an insurance company. Moreover, we have shown in Section 5 that some serious underestimations may occur if linear correlations are used for measuring dependencies and that other dependency ratios should be preferred.

Table 1. Correlation matrix for aggregating risk modules in Solvency II.

\begin{tabular}{|c|c|c|c|c|c|}
\hline$\rho_{i, j}$ & $j=1:$ market risk & $\begin{array}{c}j=2: \text { counterparty } \\
\text { default risk }\end{array}$ & $\begin{array}{l}\qquad j=3 \text { : life } \\
\text { underwriting risk }\end{array}$ & $\begin{array}{l}\quad j=4 \text { : health } \\
\text { underwriting risk }\end{array}$ & $\begin{array}{c}j=5 \text { : non-life } \\
\text { underwriting risk }\end{array}$ \\
\hline$i=1:$ market risk & 1 & 0.25 & 0.25 & 0.25 & 0.25 \\
\hline$i=2:$ counterparty default risk & 0.25 & 1 & 0.25 & 0.25 & 0.5 \\
\hline$i=3$ : life underwriting risk & 0.25 & 0.25 & 1 & 0.25 & 0 \\
\hline$i=4$ : health underwriting risk & 0.25 & 0.25 & 0.25 & 1 & 0 \\
\hline$i=5:$ non-life underwriting risk & 0.25 & 0.5 & 0 & 0 & 1 \\
\hline
\end{tabular}


However, the Solvency II framework gives the possibility to apply also the concept of copulas to capture dependencies in an internal model. The supervisory authorities may even require the companies to apply an internal model for calculating the Solvency Capital Requirement, or a part thereof, if it is inappropriate to calculate the Solvency Capital Requirement using the standard approach [1]. That means that if the approach for considering dependencies that is given in the standard model does not lead to a realistic picture of the actual risk situation of the company, the supervisory authorities may oblige the company to use a more sophisticated way for capturing dependencies.

Since the Solvency II framework does not use copulas in the standard formula for reason of the proportionality principle, we recommend that Solvency II should at least reward those insurers which measure the dependencies between their risks in a more sophisticated way. This could be achieved either by reducing the SCR for those insurers or the other way around by imposing higher requirements on companies which use the rudimental standard approach. That would also be justifiable from an economical point of view: Companies that use linear correlations may severely underestimate their overall risk and should therefore be protected by higher capital requirements.

It would also make sense and give additional incentives to explicitly mention the concept of copulas in the directive and to rework the standard formula once the development in multivariate modeling allows the effective use of copulas also for smaller insurance companies [20]. Moreover, we have discovered that the given correlations do not seem to reflect an actual average of the insurance industry. So, if correlations are used, they should at least be actually measured in the insurance industry.

\section{References}

[1] European Commission, "Directive of the European Parliament and of the Council on the Taking-Up and Pursuit of the Business of Insurance and Reinsurance," 2009. http://register.consilium.europa.eu/pdf/en/09/st03/ st03643-re06.en09.pdf

[2] S. Wang, "Aggregation of Correlated Risk Portfolios: Models and Algorithms," Proceedings of the Casualty Actuarial Society, Vol. 85, 1998, pp. 848-939.

[3] E. W. Frees and E. A. Valdez, "Understanding Relationships Using Copulas," North American Actuarial Journal, Vol. 2, No. 1, 1998, pp. 1-25.

[4] P. Blum, A. Dias and P. Embrechts, "The ART of Dependence Modeling: The Latest Advances in Correlation Analysis," In: M. Lane, Ed., Alternative Risk Strategies, Risk Books, London, 2002.

[5] A. J. McNeil, "Sampling Nested Archimedean Copulas," Journal of Statistical Computation and Simulation, Vol.
78, No. 6, 2007, pp. 567-581. doi:10.1080/00949650701255834

[6] M. Eling and D. Toplek, "Modeling and Management of Nonlinear Dependencies-Copulas in Dynamic Financial Analysis," Journal of Risk and Insurance, Vol. 76, No. 3, 2009, pp. 651-681. doi:10.1111/j.1539-6975.2009.01318.x

[7] A. Tang and E. A. Valdez, "Economic Capital and the Aggregation of Risks using Copulas," 2006. http://www.ica2006.com/Papiers/282/282.pdf

[8] A. Patton, "Copula-Based Models for Financial Time Series,” In: T. G. Andersen, R. A. Davies, J.-P. Kreiss and T. Mikosch, Ed., Handbook of Financial Time Series, Springer, Berlin, 2009, pp. 767-785. doi:10.1007/978-3-540-71297-8 34

[9] C. Genest, M. Gendron and M. Bourdeau-Brien, "The Advent of Copulas," European Journal of Finance, Vol. 15, No. 7-8, 2009, pp. 609-618. doi:10.1080/13518470802604457

[10] G. Szegö, "Measures of Risk," Journal of Banking and Finance, Vol. 26, No. 7, 2002, pp. 1253-1272. doi:10.1016/S0378-4266(02)00262-5

[11] D. Pfeifer, "Möglichkeiten und Grenzen der mathematischen Schadenmodellierung," Zeitschrift für die gesamte Versicherungswissenschaft-German Journal of Risk and Insurance, Vol. 92, No. 4, 2003, pp. 665-696.

[12] M. Sklar, "Fonctions de Répartition à n Dimensions et Leurs Marges," Publications de l'Institut de Statistique de l'Université de Paris, No. 8, 1959, pp. 229-231.

[13] P. Embrechts, A. McNeil and D. Straumann, "Correlation and Dependence in Risk Management: Properties and Pitfalls," 2002.

http://www.math.ethz.ch/ strauman/ preprints/pitfalls.pdf

[14] H.-J. Zwiesler, "Asset-Liability-Management-Die Versizcherung auf dem Weg von der Planungsrechnung zum Risikomanagement," In: K. Spremann, Ed., Versicherungen im Umbruch-Werte Schaffen, Risiken managen, Kunden gewinnen, Springer, Berlin, 2005, pp. 117-131.

[15] T. Ané, T. and C. Kharoubi, "Dependence Structure and Risk Measure," Journal of Business, Vol. 76, No. 3, 2003, pp. 411-438. doi:10.1086/375253

[16] M. Junker and A. May, "Measurement of aggregate risk with copulas," Econometrics Journal, Vol. 8, No. 3, 2005, pp. 428-454. doi:10.1111/j.1368-423X.2005.00173.x

[17] G. G. Venter, "Tails of Copulas," Proceedings of the Casualty Actuarial Society, Vol. 89, No. 171, 2002, pp. 68-113.

[18] C. Genest and L.-P. Rivest, "Statistical Inference Procedures for Bivariate Archimedean Copulas," Journal of the American Statistical Association, Vol. 88, No. 423, 1993, pp. 1034-1043. doi:10.2307/2290796

[19] E. W. Frees and E. A. Valdez, "Understanding Relationships Using Copulas," North American Actuarial Journal, Vol. 2, No. 1, 1998, pp. 1-25.

[20] P. Embrechts, "Copulas: A Personal View," Journal of Risk and Insurance, Vol. 76, No. 3, 2009, pp. 639-650. doi:10.1111/j.1539-6975.2009.01310.x 